\title{
The potential role of kinesin and dynein in Golgi scattering and cytoplasmic vacuole formation during acute experimental pancreatitis
}

Cell Research (2010) 20:599-602. doi:10.1038/cr.2010.42; published online 6 April 2010

\section{Dear Editor,}

Acute pancreatitis is initiated in pancreatic acinar cells and characterized by a profound disturbance in intracellular vesicle transport. Moreover, secretion of pancreatic zymogens is blocked, paralleled by the formation of cytoplasmic vacuoles with premature protease activation that precedes cellular necrosis and organ damage [1]. An early event triggering the development of acute pancreatitis could be the rapid disassembly of the acinar cell cytoskeleton that has been observed during secretagogueinduced pancreatitis in rodents [2]. Microtubules and their motor proteins have been shown to be critically involved in vesicular trafficking and polarized secretion [3]. Furthermore, microtubule-dependent mechanisms seem to be responsible for the replenishment of zymogen granules after secretion, presumably by mediating the movement of Golgi vesicles toward the acinar cell lumen [4]. Impairment of these mechanisms early during pancreatitis could therefore lead to a block in secretion and to the formation of cytoplasmic vacuoles, in which premature activation of zymogens occurs. This is supported by the observation that treatment with compounds blocking cytoskeletal function led to intracellular perturbations similar to those observed during acute pancreatitis [5], and that pharmacological prevention of microtubule dissociation prevented acute pancreatitis in rodents [6].

In the present study, we used the rat model of caerulein-induced pancreatitis to investigate the role of microtubule motor proteins kinesin and dynein in the development of acute pancreatitis. We first assessed the expression of kinesin, dynein and tubulin during experimental pancreatitis. To this end, pancreatic homogenates were prepared from male Wistar rats, anesthetized with pentobarbital sodium $(60 \mathrm{mg} / \mathrm{kg})$ during surgery and infused with supramaximal concentrations of caerulein

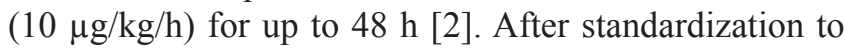
DNA content, western blot analysis was performed with antibodies raised against kinesin-1 heavy chain and cy- toplasmic dynein $74 \mathrm{kD}$ intermediate chain, as described previously [4]. As shown in Figure 1A, protein levels of tubulin, dynein and kinesin in the pancreas barely change during the early stages of pancreatitis, suggesting that proteolytic degradation of motor proteins is not the cause for intracellular trafficking disorders. After $1 \mathrm{~h}$ of caerulein administration, tubulin and kinesin signal intensities were not altered, whereas a decrease in dynein signal intensity and an additional dynein signal at a lower molecular weight were visible during the acute phase of pancreatitis, indicating that dynein was partially degraded during pancreatitis.

To determine whether the association of tubulin with its molecular motors changed during the initial phase of pancreatitis, we purified microtubules from pancreatic tissue at different time points of caerulein administration using a method based on temperature-dependent microtubule disassembly/reassembly [4]. Purified microtubule fractions displayed similar signal intensities of dynein, kinesin and tubulin (Figure 1B), suggesting that microtubule motor protein association is not affected during the whole course of experimental pancreatitis.

We further examined the intracellular distribution of kinesin and dynein during caerulein-induced pancreatitis by immunostaining of pancreatic sections with anti-kinesin and anti-dynein monoclonal antibodies [7]. Remarkably, dynein and kinesin were predominantly localized to the perinuclear region of acinar cells and did not relocate during the whole course of pancreatitis (Figure 1C and 1D), suggesting that they are not directly involved in the impairment of apical secretion.

Impairment of microtubule-dependent mechanisms at the early stages of pancreatitis could lead to a disorganization of the Golgi complex in pancreatic acinar cells [8]. The Golgi apparatus of pancreatic acinar cells has been described in detail [9]. We analyzed Golgi morphology by immunofluorescence microscopy of pancreatic sections obtained after different time points of caerulein administration using antibodies directed against the Golgi 
A

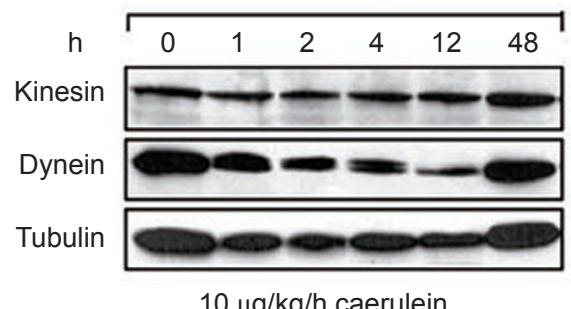

C
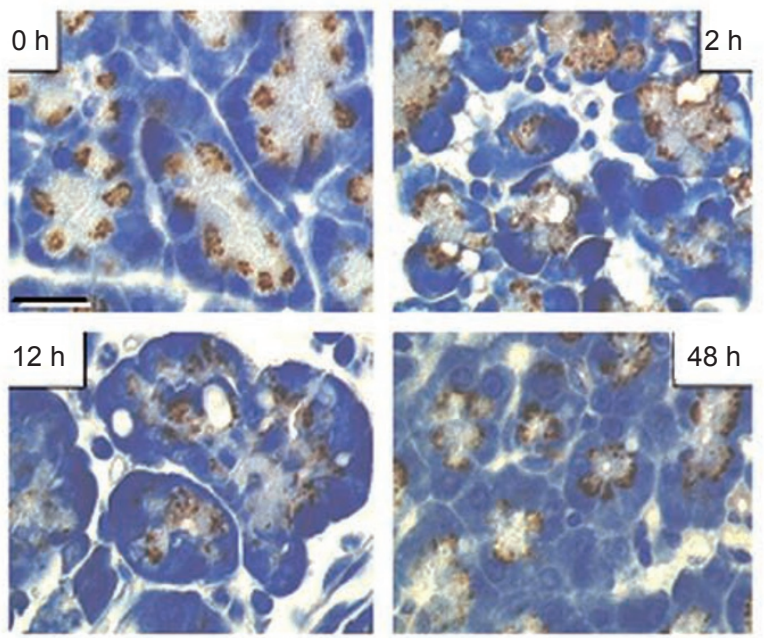

B

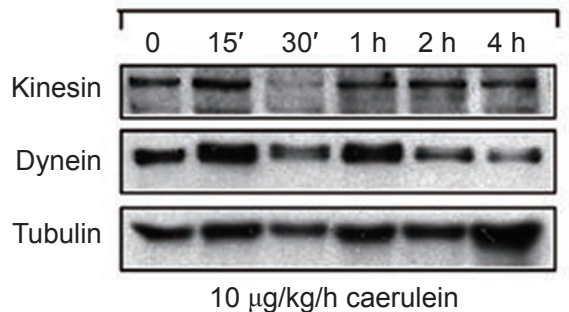

D
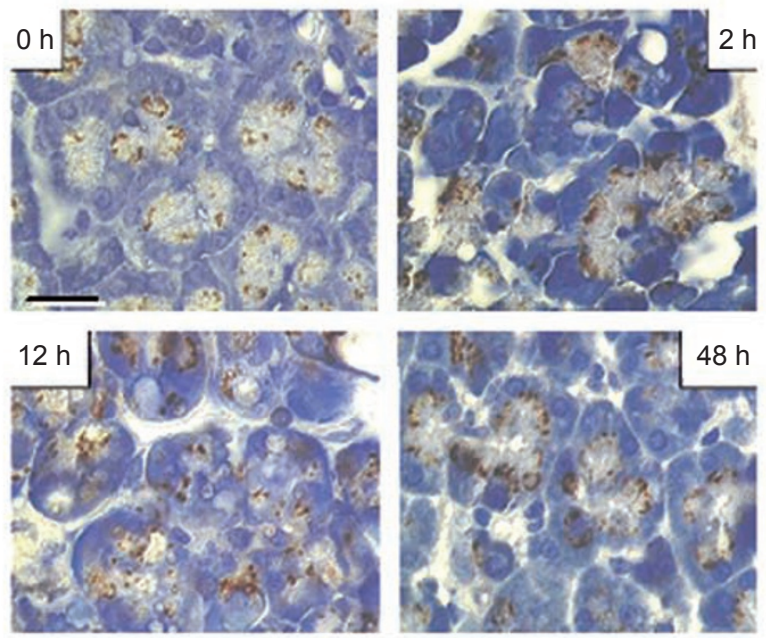

E
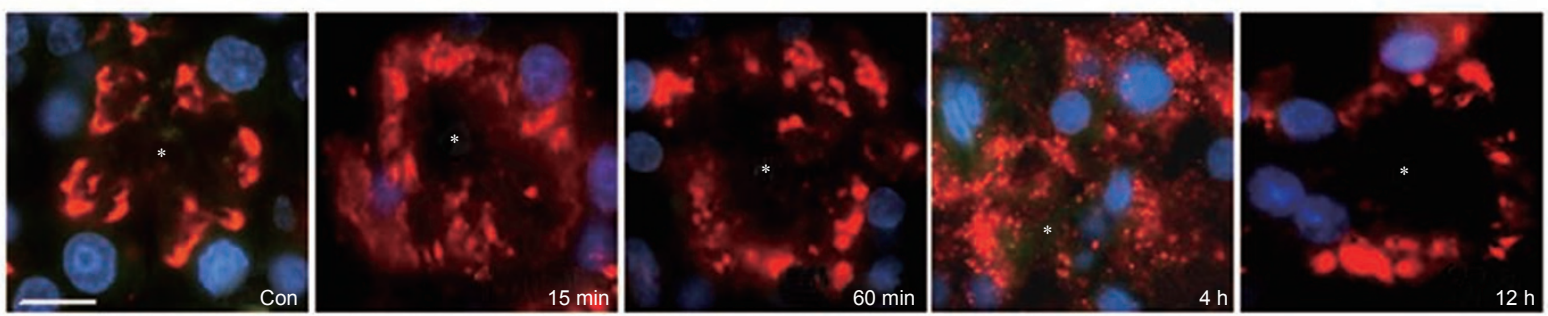

F

Dynein

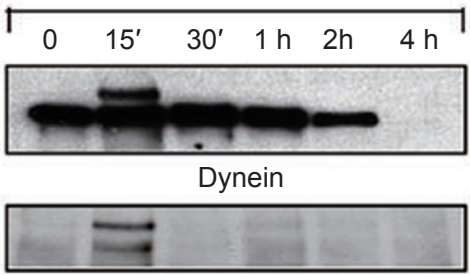

Phosphotyrosine
Kinesin

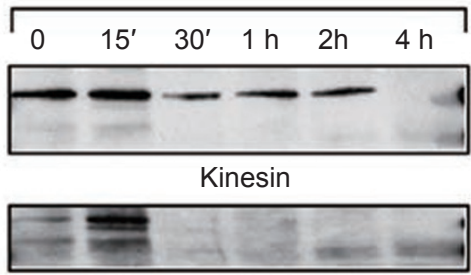

Phosphotyrosine 
marker 58K (for details see Schnekenburger et al. [4]). During the initial and acute phase of pancreatitis (15 min to $4 \mathrm{~h}$ ), the Golgi complex was dispersed throughout the acinar cell and displayed a vesiculated appearance, whereas at $12 \mathrm{~h}$ after caerulein treatment, the Golgi signal was redistributed to the perinuclear region (Figure 1E).

Since various studies demonstrated that kinesin and dynein are critically involved in Golgi positioning and function (for review see Allan et al. [10]), Golgi scattering could be the result of a dissociation of motor proteins from the Golgi complex. Furthermore, it has been suggested that phosphorylation of kinesin and dynein plays a negative role in regulating cargo binding $[11,12]$. We therefore determined whether kinesin and dynein were phosphorylated during caerulein-induced pancreatitis. Western blots prepared with immunoprecipitates of kinesin and dynein from pancreatic homogenates were analyzed using monoclonal anti-phosphotyrosine antibodies [4]. As shown in Figure 1F, both molecular motors undergo tyrosine phosphorylation within $15 \mathrm{~min}$ of the onset of experimental pancreatitis, but do not appear to be tyrosine phosphorylated later than $30 \mathrm{~min}$ after caerulein treatment. Kinesin and dynein might therefore be involved in the concurrent scattering of the Golgi complex, thereby contributing to a derangement of intracellular enzyme sorting and to the formation of poorly condensed vacuoles associated with acute pancreatitis [13].

Taken together, these data and prior studies suggest that Golgi fragmentation is one of the initiating events in acute pancreatitis, that it is paralleled by tyrosine phosphorylation of dynein and kinesin and that its consequence consists of the formation of cytoplasmic vacuoles. Whether these vacuoles represent the principle site of premature intracellular zymogen activation - and thus the triggering event of pancreatitis - or merely contribute to an arsenal of potential activation organelles will have to be explored in future studies.

\section{Acknowledgments}

This work was supported by grants from the Deutsche Forschungsgemeinschaft (Le 625/8-1 and 9-1 to MML, SFB 293 B7 to JS and MML), the Alfred Krupp Foundation and BMBF (KF0409001MD6), as well as the IZKF Münster (IZKF H3 to JS and MML). We wish to thank U Breite, H Heitmann, the Department of Medicine B, Westfälische Wilhelms-University and V Samoilova, the Gerhard Domagk Institute of Pathology, Westfälische Wilhelms-University, for expert technical assistance.

Ina A Weber ${ }^{1}$, Igor Buchwalow ${ }^{2}$, Daniela Hahn ${ }^{1}$, Wolfram Domschke ${ }^{1}$, Markus M Lerch ${ }^{3, *}$, Jürgen Schnekenburger ${ }^{1, *}$

${ }^{I}$ Department of Medicine B, Westfälische Wilhelms-University, D-48149 Münster, Germany; ${ }^{2}$ Gerhard Domagk Institute of Pathology, Westfälische Wilhelms-University, D-48149 Münster, Germany; ${ }^{3}$ Department of Medicine A, Ernst-Moritz-ArndtUniversity, D-17487 Greifswald, Germany

*These two authors contributed equally to this work. Correspondence: Jürgen Schnekenburger

Tel: +49 25183 52534; Fax: +49 2518357938

E-mail: schnekenburger@uni-muenster.de

\section{References}

1 Saluja AK, Lerch MM, Phillips PA, Dudeja V. Why does pancreatic overstimulation cause pancreatitis? Annu Rev Physiol 2007; 69:249-269.

2 Jungermann J, Lerch MM, Weidenbach H, Lutz MP, Krüger B, Adler G. Disassembly of rat pancreatic acinar cell cytoskeleton during supramaximal secretagogue stimulation. Am J Physiol

Figure 1 (A) Western blot of tubulin, dynein and kinesin in pancreatic homogenates obtained after treatment with saline $(0 \mathrm{~h})$ or with caerulein $(10 \mu \mathrm{g} / \mathrm{kg} / \mathrm{h})$ for the time period indicated. DNA content of pancreatic homogenates was used as a standard for loading equal amounts of pancreatic protein on SDS gels. (B) Association of kinesin and dynein with tubulin. Tubulin and associated motor proteins were purified from homogenates obtained after infusion of rats with either saline or caerulein $(10 \mu \mathrm{g} / \mathrm{kg} / \mathrm{h})$ by two cycles of temperature-dependent disassembly/reassembly [4]. Equal amounts of protein were used for western blot analysis with monoclonal antibodies raised against tubulin, kinesin and dynein. (C) Immunostaining of dynein. Pancreatic paraffin sections from rats infused with either saline $(0 \mathrm{~h})$ or caerulein $(10 \mu \mathrm{g} / \mathrm{kg} / \mathrm{h})$ for the time intervals indicated were labeled with monoclonal antibodies raised against dynein intermediate chain, biotinylated secondary antibodies and with a complex of avidin and biotinylated horseradish peroxidase. Tissue was stained with hematoxylin and eosin. Note the signs of acute pancreatitis such as vacuolization, edema formation and widening of the interstitial space at $2 \mathrm{~h}$ and $12 \mathrm{~h}$. (D) Immunostaining of kinesin with monoclonal anti-kinesin antibodies. For further details, see C. (E) Immunofluorescence microscopy of the Golgi marker 58K. Pancreatic paraffin sections from rats infused with either saline (Con) or caerulein (10 $\mu \mathrm{g} / \mathrm{kg} / \mathrm{h}$ ) for the time intervals indicated were labeled using anti-Golgi 58K monoclonal antibodies and Cy3-marked secondary antibodies. Nuclei were counterstained with DAPI and appear in blue. Asterisks denote the acinar lumen. Scale bar, $10 \mu \mathrm{m}$. (F) Western blot analysis of kinesin and dynein immunoprecipitates with anti-kinesin, anti-dynein and anti-phosphotyrosine antibodies. Precipitates were obtained from pancreatic homogenates from rats after treatment with either saline $(0 \mathrm{~h})$ or caerulein $(10 \mu \mathrm{g} / \mathrm{kg} / \mathrm{h})$ for the time intervals indicated. All experiments were repeated using at least three animals per time point. 
1995; 268 (2 Pt 1):G328-G338.

3 Hehnly H, Stamnes M. Regulating cytoskeleton-based vesicle motility. FEBS Lett 2007; 581:2112-2118.

4 Schnekenburger J, Weber IA, Hahn D, et al. The role of kinesin, dynein and microtubules in pancreatic secretion. Cell Mol Life Sci 2009; 66:2525-2537.

5 Williams JA, Lee M. Microtubules and pancreatic amylase release by mouse pancreas in vitro. J Cell Biol 1976; 71:795806.

6 Ueda T, Takeyama Y, Kaneda K, Adachi M, Ohyanagi H, Saitoh Y. Protective effect of a microtubule stabilizer taxol on caerulein-induced acute pancreatitis in rat. J Clin Invest 1992; 89:234-243.

7 Schnekenburger J, Schick V, Kruger B, et al. The calcium binding protein S100A9 is essential for pancreatic leukocyte infiltration and induces disruption of cell-cell contacts. $J$ Cell Physiol 2008; 216:558-567.
8 Pavelka M, Ellinger A. Effect of colchicine on the Golgi complex of rat pancreatic acinar cells. J Cell Biol 1983; 97:737748.

9 Palade G. Intracellular aspects of the process of protein synthesis. Science 1975; 189:347-358.

10 Allan VJ, Thompson HM, McNiven MA. Motoring around the Golgi. Nat Cell Biol 2002; 4:E236-E242.

11 Sato-Yoshitake R, Yorifuji H, Inagaki M, Hirokawa N. The phosphorylation of kinesin regulates its binding to synaptic vesicles. J Biol Chem 1992; 267:23930-23936.

12 Vaughan PS, Leszyk JD, Vaughan KT. Cytoplasmic dynein intermediate chain phosphorylation regulates binding to dynactin. J Biol Chem 2001; 276:26171-26179.

13 Van Acker GJ, Weiss E, Steer ML, Perides G. Cause-effect relationships between zymogen activation and other early events in secretagogue-induced acute pancreatitis. Am J Physiol 2007; 292:G1738-G1746. 